\title{
CONNECTED DOMINATION NUMBER OF A GRAPH AND ITS COMPLEMENT
}

\author{
H. KARAMI AND S.M. SHEIKHOLESLAMI* \\ DEPARTMENT OF MATHEMATICS \\ AZARBAIJAN UNIVERSITY OF TARBIAT MOALLEM \\ TABRIZ, I.R. IRAN \\ S.M.SHEIKHOLESLAMI@AZARUNIV.EDU \\ ABDOLLAH KHODKAR \\ DEPARTMENT OF MATHEMATICS \\ UNIVERSITY OF WEST GEORGIA \\ CARROLLTON, GA 30118 \\ AKHODKAR@WESTGA .EDU \\ DOUGLAS B. WEST ${ }^{\dagger}$ \\ MATHEMATICS DEPARTMENT \\ UNIVERSITY OF ILLINOIS \\ URBANA, IL 61801 \\ WEST@MATH . UIUC.EDU
}

\begin{abstract}
A set $S$ of vertices in a graph $G$ is a connected dominating set if every vertex not in $S$ is adjacent to some vertex in $S$ and the subgraph induced by $S$ is connected. The connected domination number $\gamma_{c}(G)$ is the minimum size of such a set. Let $\delta^{*}(G)=\min \{\delta(G), \delta(\bar{G})\}$, where $\bar{G}$ is the complement of $G$ and $\delta(G)$ is the minimum vertex degree. We prove that when $G$ and $\bar{G}$ are both connected, $\gamma_{c}(G)+\gamma_{c}(\bar{G}) \leq \delta^{*}(G)+4-\left(\gamma_{c}(G)-3\right)\left(\gamma_{c}(\bar{G})-3\right)$. As a corollary, $\gamma_{c}(G)+\gamma_{c}(\bar{G}) \leq \frac{3 n}{4}$ when $\delta^{*}(G) \geq 3$ and $n \geq 14$, where $G$ has $n$ vertices. We also prove that $\gamma_{c}(G)+\gamma_{c}(\bar{G}) \leq \delta^{*}(G)+2$ when $\gamma_{c}(G), \gamma_{c}(\bar{G}) \geq 4$. This bound is sharp when $\delta^{*}(G)=6$, and equality can only hold when $\delta^{*}(G)=6$. Finally, we prove that $\gamma_{c}(G) \gamma_{c}(\bar{G}) \leq 2 n-4$ when $n \geq 7$, with equality only for paths and cycles.
\end{abstract}

Keywords: connected dominating set, connected domination number, NordhausGaddum inequalities.

MSC 2000: 05C69

\section{INTRODUCTION}

Many problems in extremal graph theory seek the extreme values of graph parameters on families of graphs. Results of Nordhaus-Gaddum type study the extreme values of the sum (or product) of a parameter on a graph and its complement, following the classic paper of Nordhaus and Gaddum [8] solving these problems for the

* Corresponding author.

$\dagger$ This research partially supported by the National Security Agency under Awards H98230-06-10065 and H98230-10-1-0363. 
chromatic number on $n$-vertex graphs. In this paper, we study such problems for the connected domination number.

For domination problems, multiple edges and loops are irrelevant, so we forbid them. We use $V(G)$ and $E(G)$ for the vertex set and edge set of a graph $G$. For a vertex $v \in V(G)$, the open neighborhood $N(v)$ is the set $\{u \in V(G): u v \in E(G)\}$ and the closed neighborhood $N[v]$ is the set $N(v) \cup\{v\}$. The open neighborhood $N(S)$ of a set $S \subseteq V$ is the set $\bigcup_{v \in S} N(v)$, and the closed neighborhood $N[S]$ of $S$ is the set $N(S) \cup S$. The minimum and maximum vertex degrees in $G$ are denoted $\delta(G)$ and $\Delta(G)$, respectively. Given graphs $G$ and $H$, the cartesian product $G \square H$ is the graph with vertex set $V(G) \times V(H)$ and edge set defined by making $(u, v)$ and $\left(u^{\prime}, v^{\prime}\right)$ adjacent if and only if either (1) $u=u^{\prime}$ and $v v^{\prime} \in E(H)$ or $(2) v=v^{\prime}$ and $u u^{\prime} \in E(G)$.

For a graph $G$, a set $S \subseteq V(G)$ is a dominating set if $N[S]=V(G)$, and $S$ is a connected dominating set if also the subgraph induced by $S$, denoted $G[S]$, is connected. The minimum size of a dominating set and a connected dominating set are the domination number $\gamma(G)$ and the connected domination number $\gamma_{c}(G)$, respectively.

Inequalities of Nordhaus-Gaddum type have been proved for many variations of domination parameters. Some of them can be improved when constraints on $G$ and $\bar{G}$ are imposed. For the original domination number itself, the following bounds have been proved.

(1) $\gamma(G)+\gamma(\bar{G}) \leq n+1$ for every graph $G[6]$;

(2) $\gamma(G)+\gamma(\bar{G}) \leq \frac{n}{2}+2$ if $\delta(G), \delta(\bar{G}) \geq 1[5]$;

(3) $\gamma(G)+\gamma(\bar{G}) \leq \frac{2 n}{5}+3$ if $\delta(G), \delta(\bar{G}) \geq 2$, with some small exceptions [2];

(4) $\gamma(G)+\gamma(\bar{G}) \leq \frac{3 n}{8}+2$ if $\delta(G), \delta(\bar{G}) \geq 3$, with some small exceptions [2].

Throughout this paper we impose the following condition: $G$ is a connected $n$ vertex graph whose complement $\bar{G}$ is also connected. Note that this requires $n \geq 4$. For such $G$, we establish sharp upper bounds for $\gamma_{c}(G)+\gamma_{c}(\bar{G})$ and $\gamma_{c}(G) \cdot \gamma_{c}(\bar{G})$ in terms of $n$ and the minimum degrees of $G$ and $\bar{G}$. In the list below of our results, (1) is our main result, and most of the others follow from closer examination of its proof. Let $\delta^{*}(G)=\min \{\delta(G), \delta(\bar{G})\}$.

(1) $\gamma_{c}(G)+\gamma_{c}(\bar{G}) \leq \delta^{*}(G)+4-\left(\gamma_{c}(G)-3\right)\left(\gamma_{c}(\bar{G})-3\right)$; sharp for $\delta^{*}(G) \geq 2$.

(2) $\gamma_{c}(G)+\gamma_{c}(\bar{G}) \leq \frac{3 n}{4}$ when $\delta^{*}(G) \geq 3$ and $n \geq 14$; sharp when 4 divides $n$.

(3) $\gamma_{c}(G)+\gamma_{c}(\bar{G}) \leq \delta^{*}(G)+2$ when $\gamma_{c}(G), \gamma_{c}(\bar{G}) \geq 4$, with equality possible if and only if $\delta^{*}(G)=6$.

(4) $\gamma_{c}(G) \gamma_{c}(\bar{G}) \leq 2 n-4$ when $n \geq 7$, with equality only when $G$ or $\bar{G}$ is a path or cycle.

If $G$ or $\bar{G}$ has a dominating vertex, then its complement is disconnected, so we may assume that $\gamma_{c}(G), \gamma_{c}(\bar{G}) \geq 2$. When $\gamma_{c}(\bar{G})=2$, our first inequality reduces to $2 \leq \delta^{*}(G)+1$, which holds since both graphs are connected. Hence we may assume that $\gamma_{c}(G), \gamma_{c}(\bar{G}) \geq 3$. This condition is equivalent to $\operatorname{diam} G=\operatorname{diam} \bar{G}=2$, since it is immediate from the definitions that $\operatorname{diam} G \geq 3$ if and only if $\gamma_{c}(\bar{G}) \leq 2$.

We note first that when $\gamma_{c}(\bar{G})=2$, the best bound on the sum is $\gamma_{c}(G)+\gamma_{c}(\bar{G}) \leq n$. The existence of a spanning tree (with at least two leaves) in a connected graph yields $\gamma_{c}(G) \leq n-2$; equality holds for paths and cycles. (The fact that $\gamma_{c}(G)=n-\ell(G)$, where $\ell(G)$ is the maximum number of leaves in a spanning tree, was first noted by 
Hedetniemi and Laskar in [4]). Restricting the problem in the case $\gamma_{c}(\bar{G})=2$ by requiring also $\delta(G) \geq 3$ leads to $\gamma_{c}(G)+\gamma_{c}(\bar{G}) \leq \frac{3 n}{4}$, by Theorem A below.

Theorem A. [3, 7] If $G$ is a connected $n$-vertex graph and $\delta(G) \geq k$, where $k \leq 5$, then $\gamma_{c}(G) \leq \frac{3 n}{k+1}-c_{k}$, where $c_{k}$ is a small constant (in particular, $c_{3}=2$ and $\left.c_{4}=8 / 5\right)$.

The case $k=3$ of Theorem A was proved independently by many researchers. Further increases in $\delta(G)$ lead to further reductions in $\gamma_{c}(G)$ and hence also in the bound on $\gamma_{c}(G)+\gamma_{c}(\bar{G})$ when $\gamma_{c}(\bar{G})=2($ see $[1,7])$.

It is well known that in every connected graph $G$ there is a spanning tree with at least $\Delta(G)$ leaves. This yields another remark from [4] that we will find useful.

Theorem B. [4] If $G$ is a connected $n$-vertex graph, then $\gamma_{c}(G) \leq n-\Delta(G)$.

\section{Bounds ON $\gamma_{c}(G)+\gamma_{c}(\bar{G})$}

In this section we establish sharp upper bounds on the sum $\gamma_{c}(G)+\gamma_{c}(\bar{G})$ in terms of the number of vertices and the minimum degrees of $G$ and $\bar{G}$.

Theorem 1. If $G$ and $\bar{G}$ are connected, then

$$
\gamma_{c}(G)+\gamma_{c}(\bar{G}) \leq \delta^{*}(G)+4-\left(\gamma_{c}(G)-3\right)\left(\gamma_{c}(\bar{G})-3\right) .
$$

Proof. As noted in the Introduction, we may assume that $\gamma_{c}(G), \gamma_{c}(\bar{G}) \geq 3$ and $\operatorname{diam}(G)=\operatorname{diam}(\bar{G})=2$. Let $x$ be a vertex of degree $\delta(G)$, and let $X=V(G)-N[x]$. Since $\gamma_{c}(G) \geq 3$, we have $X \neq \emptyset$. Also, $N(x)$ dominates $X$, since $\operatorname{diam}(G)=2$.

We successively select disjoint sets $S_{0}, \ldots, S_{k}$ in $N(x)$ that almost dominate $X$. Let $T_{0}=N(x)$, and let $S_{0}$ be a largest subset of $N(x)$ that does not dominate $X$. Let $T_{1}=T_{0}-S_{0}$. By the maximality of $S_{0}$, every vertex $t$ of $T_{1}$ dominates $X-N\left(S_{0}\right)$, but $T_{1}$ may or may not dominate $X$. We continue, constructing sets $T_{0}, \ldots, T_{k}$ with $T_{0} \supset \ldots \supset T_{k}($ where $k \geq 1)$ and sets $S_{0}, \ldots S_{k-1}$ such that

(a) For $i<k$, the set $T_{i}$ dominates $X$.

(b) For $i<k$, the set $S_{i}$ is a largest subset of $T_{i}$ not dominating $X$, and $T_{i+1}=T_{i}-S_{i}$.

(c) $T_{k}$ does not dominate $X$.

Since $T_{i}$ dominates $X$ but $S_{i}$ does not (when $i<k$ ), all of $T_{0}, \ldots, T_{k}$ are nonempty. By construction, $S_{i} \cup\{y\}$ dominates $X$ whenever $y \in T_{i+1}$. Thus $S_{i} \cup\{x, y\}$ is a connected dominating set, so $\left|S_{i}\right| \geq \gamma_{c}(G)-2$. For $0 \leq i \leq k-1$, let $x_{i}$ be a vertex of $X$ that is not dominated by $S_{i}$, and let $x_{k}$ be a vertex of $X$ that is not dominated by $T_{k}$. Since $N(x)=\left(\cup S_{i}\right) \cup T_{k}$, the set $\left\{x, x_{0}, \ldots, x_{k}\right\}$ is a connected dominating set of $\bar{G}$, so $k \geq \gamma_{c}(\bar{G})-2$. Since $\left|S_{0}\right|=\delta(G)-\left|T_{k}\right|-\sum_{i=1}^{k-1}\left|S_{i}\right|$ and $\left|T_{k}\right| \geq 1$,

$$
\begin{aligned}
\gamma_{c}(G)+\gamma_{c}(\bar{G}) & \leq\left(\left|S_{0}\right|+2\right)+(k+2) \\
& =\left(\delta(G)-\left|T_{k}\right|-\sum_{i=1}^{k-1}\left|S_{i}\right|+2\right)+(k+2) \\
\leq & \delta(G)-\left|T_{k}\right|+4-(k-1)\left(\gamma_{c}(G)-2\right)+k \\
\leq & \delta(G)+4-(k-1)\left(\gamma_{c}(G)-3\right) \\
& \leq \delta(G)+4-\left(\gamma_{c}(\bar{G})-3\right)\left(\gamma_{c}(G)-3\right) .
\end{aligned}
$$

By symmetry, $\gamma_{c}(G)+\gamma_{c}(\bar{G}) \leq \delta(\bar{G})+4-\left(\gamma_{c}(G)-3\right)\left(\gamma_{c}(\bar{G})-3\right)$. 
Theorem 2. The bound of Theorem 1 is sharp for each value of $\delta^{*}(G)$ at least 2 .

Proof. For each integer $r$ with $r \geq 2$, we construct a connected graph $G_{r}$ with $\delta\left(G_{r}\right)=$ $r<\delta\left(\bar{G}_{r}\right), \gamma_{c}(\bar{G})=3$, and $\gamma_{c}\left(G_{r}\right)+\gamma_{c}\left(\bar{G}_{r}\right)=r+4$, thereby achieving the bound. The graph $G_{r}$ will have $r^{2}+r+1$ vertices, with $\gamma_{c}\left(\bar{G}_{r}\right)$ kept small by making $\delta\left(\bar{G}_{r}\right)$ large: $\delta\left(\bar{G}_{r}\right)=r^{2}-r+1$.

Form the graph $G_{r}$ as follows. Let $H_{1}=H_{2}=K_{r}$, with $V\left(H_{2}\right)=\left\{v_{1}, \ldots, v_{r}\right\}$. To the cartesian product $H_{1} \square H_{2}$, add a star with $r+1$ new vertices, having center $y$ and leaves $x_{1}, \ldots, x_{r}$. For $1 \leq i \leq r$, add edges joining $x_{i}$ to all vertices of $H_{1} \square H_{2}$ with second coordinate $v_{i}$. The resulting graph is $G_{r}$; Figure 1 shows $G_{2}$ (along with $H_{1}$ and $\left.H_{2}\right)$. Note that $\operatorname{diam}\left(G_{r}\right)=\operatorname{diam}\left(\bar{G}_{r}\right)=2$ and that $\delta^{*}\left(G_{r}\right)=r$; the degrees in $G$ of $y, x_{i}$ and vertices of $H_{1} \square H_{2}$ are $r, r+1$, and $2 r-1$, respectively.

It suffices to show that $\gamma_{c}\left(\bar{G}_{r}\right)=3$ and $\gamma_{c}\left(G_{r}\right)=r+1$. Since $\operatorname{diam}(G)=2$, we have $\gamma_{c}(\bar{G}) \geq 3$. Equality holds using $\{y, u, w\}$, where $u$ and $w$ are neighbors of $x_{1}$ and $x_{2}$ in $G$ other than $y$.

To see that $\gamma_{c}\left(G_{r}\right)=r+1$, note first that $\left\{y, x_{1}, \ldots, x_{r}\right\}$ is a connected dominating set. For the lower bound, let $S$ be a connected dominating set, and let $T_{i}=N\left[x_{i}\right]-\{y\}$. If $S$ does not intersect $T_{i}$, which includes $x_{i}$ and a copy of $V\left(H_{1}\right)$, then dominating $T_{i}$ requires $S$ to contain $y$ and a vertex in each copy of $H_{2}$. This requires $r+1$ vertices. Thus $|S| \geq r+1$ unless $S$ intersects each of the $r$ disjoint sets $T_{1}, \ldots, T_{r}$ exactly once. Dominating $y$ without reaching size $r+1$ requires $S$ to contain some $x_{i}$, but now $x_{i}$ has no neighbor in $S$. We conclude that $\gamma_{c}(G)=r+1$.

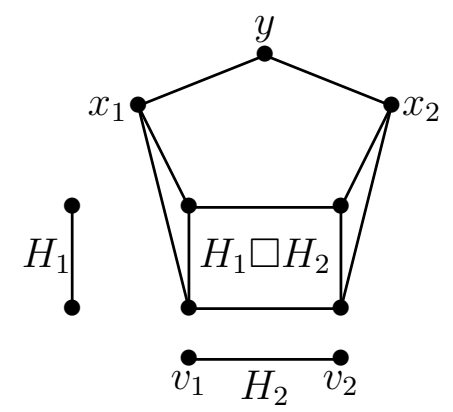

Figure 1. The graph $G_{2}$, plus $H_{1}$ and $H_{2}$

Since each vertex has $n-1$ neighbors in $G$ and $\bar{G}$ together, always $\delta^{*}(G) \leq\left\lfloor\frac{n-1}{2}\right\rfloor$, and Theorem 1 has the following immediate consequence.

Corollary 3. If $G$ and $\bar{G}$ are connected $n$-vertex graphs with $\gamma_{c}(G), \gamma_{c}(\bar{G}) \geq 3$, then $\gamma_{c}(G)+\gamma_{c}(\bar{G}) \leq\left\lfloor\frac{n+7}{2}\right\rfloor$. The bound holds with equality when $G$ is the 5 -cycle.

Equality in Corollary 3 requires $\delta^{*}(G)=\left\lfloor\frac{n-1}{2}\right\rfloor$, so $G$ must be $\frac{n-1}{2}$-regular.

Corollary 4. If $G$ and $\bar{G}$ are connected $n$-vertex graphs with $n \geq 14$ and $\delta^{*}(G) \geq 3$, then $\gamma_{c}(G)+\gamma_{c}(\bar{G}) \leq \frac{3 n}{4}$. The bound is sharp when 4 divides $n$.

Proof. If $\gamma_{c}(G) \leq 2$ or $\gamma_{c}(\bar{G}) \leq 2$, then Theorem A applies. If $\gamma_{c}(G), \gamma_{c}(\bar{G}) \geq 3$ and $n \geq 14$, then Corollary 3 completes the proof of the bound.

To prove sharpness when 4 divides $n$ we use the "ring-of-cliques", used in the study of domination as early as [9]. Form a connected 3-regular graph by first putting $r$ copies of $K_{4}$ in a ring, then deleting one edge $x_{i} y_{i}$ from the $i$ th complete graph and 
replacing these edges with $y_{i} x_{i+1}$ for $1 \leq i \leq r$. Since no spanning tree has more than $n / 4+2$ leaves, equality holds in the bound.

By a closer look at the proof of Theorem 1, we can improve the upper bound when $\gamma_{c}(G)$ and $\gamma_{c}(\bar{G})$ are larger. The improvement does not contradict the sharpness example of Theorem 2, because $\gamma_{c}(\bar{G})=3$ in that construction.

Theorem 5. If $G$ and $\bar{G}$ are connected $n$-vertex graphs and $\gamma_{c}(G), \gamma_{c}(\bar{G}) \geq 4$, then $\gamma_{c}(G)+\gamma_{c}(\bar{G}) \leq \delta^{*}(G)+2$.

Proof. If $\gamma_{c}(G)>4$ or $\gamma_{c}(\bar{G})>4$, then the bound follows from Theorem 1, with strict inequality unless $\left\{\gamma_{c}(G), \gamma_{c}(\bar{G})\right\}=\{4,5\}$. When $\gamma_{c}(G)=\gamma_{c}(\bar{G})=4$, Theorem 1 yields $\delta^{*}(G) \geq 5$.

We show that $\gamma_{c}(G)=\gamma_{c}(\bar{G})=4$ cannot hold when $\delta^{*}(G)=5$. We may assume $\delta(G)=5$. Define $x, X$, and the sets $T_{0}, \ldots, T_{k}$ and $S_{0}, \ldots, S_{k-1}$ as in the proof of Theorem 1. Since we have assumed $\gamma_{c}(G)+\gamma_{c}(\bar{G})=\delta(G)+4-1$, equality holds throughout Equation (1). Thus $k=2,\left|T_{2}\right|=1$, and $\left|S_{1}\right|=2$. With $|N(x)|=5$, this yields $\left|S_{0}\right|=2$ and $\left|T_{1}\right|=3$. Since $S_{0}$ was chosen to be a largest subset of $N(x)$ that does not dominate $X$, any three vertices of $N(x)$ dominate $X$. Since $\gamma_{c}(G)=4$, no two vertices in $N(x)$ dominate $X$.

If $N(x)$ has a vertex $z$ with three nonneighbors in $N(x)$, then let $z^{\prime}$ be the remaining vertex in $N(x)$. Since $\left\{z, z^{\prime}\right\}$ does not dominate $X$, we may choose $y \in X$ such that $y$ is a common nonneighbor of $z$ and $z^{\prime}$. Now $\{x, y, z\}$ is a connected dominating set in $\bar{G}$, contradicting $\gamma_{c}(\bar{G})=4$.

Therefore, $\delta(H) \geq 2$, where $H=G[N(x)]$. Let $P$ be a 3 -vertex path in $H$. Since $H$ has only two more vertices, $V(P)$ dominates $H$. Since it consists of three vertices in $N(x)$, also $V(P)$ dominates $X$. Thus $V(P)$ is a connected dominating set in $G$, contradicting $\gamma_{c}(G)=4$.

We conclude that $\delta^{*}(G) \geq 6$. Since $\gamma_{c}(G)+\gamma_{c}(\bar{G})=8$, the inequality holds.

We have shown that the inequality of Theorem 5 is strict unless $\left\{\gamma_{c}(G), \gamma_{c}(\bar{G})\right\}$ is $\{4,5\}$ or both equal 4. Equality can hold when $\gamma_{c}(G)=\gamma_{c}(\bar{G})=4$ and $\delta^{*}(G)=6$.

Theorem 6. The bound of Theorem 5 is sharp when $\delta^{*}(G)=6$.

Proof. It suffices to construct a graph $G$ with $\delta^{*}(G)=6$ and $\gamma_{c}(G)=\gamma_{c}(\bar{G})=4$. To describe $G$, we introduce several auxiliary sets. Let $X=\left\{x_{1}, \ldots, x_{6}\right\}$. Let $Y$ be the family of all 4-element subsets of $X$; we write a member of $Y$ by listing the four indices or by naming the set. Let $Y^{\prime}=\{3456,1256,1234\}$, so that $Y^{\prime}$ consists of three special members among the 15 members of $Y$. Let $r=15\left(\begin{array}{c}12 \\ 2\end{array}\right)=15 \cdot 66$. For $A \in Y$, let $Z_{A}=\left\{z_{1}^{A}, \ldots, z_{r}^{A}\right\}$. For $A \in Y^{\prime}$, let $C_{1}^{A}, \ldots, C_{66}^{A}$ be a partition of $Z_{A}$ into sets of size 15. For $A \in Y^{\prime}$, let $f_{A}$ be a bijection mapping $\left\{C_{1}^{A}, \ldots, C_{66}^{A}\right\}$ to the set of unordered pairs of members of $Y-Y^{\prime}$.

Let $G$ be the graph with vertex set $X \cup\{x\} \cup\left(\cup_{A \in Y} Z_{A}\right)$ whose edges are as follows:

(1) edges $x x_{i}$ and $x_{i} x_{i+1}$ (indices modulo 6) for $1 \leq i \leq 6$.

(2) edges joining $x_{i}$ to $Z_{A}$ when $i \in A$;

(3) edges joining $z_{s}^{B}$ to $z_{t}^{B^{\prime}}$ when $s \neq t$ and $B \neq B^{\prime}$ with $B, B^{\prime} \in Y-Y^{\prime}$. 
(4) all edges joining $C_{s}^{A}$ to $Z_{B}$, where $1 \leq s \leq 66, A \in Y^{\prime}$, and $B \in Y-Y^{\prime}$, except those that would join vertices with the same subscript or would join $C_{s}^{A}$ to vertices of $Z_{B} \cup Z_{B^{\prime}}$, where $\left\{B, B^{\prime}\right\}=f_{A}\left(C_{s}^{A}\right)$.

Vertex $x$ has degree 6 in $G$; all other degrees in $G$ and $\bar{G}$ are larger. We claim that $\gamma_{c}(G)=\gamma_{c}(\bar{G})=4$. For $G$, the set $\left\{x, x_{1}, x_{2}, x_{3}\right\}$ is connected and dominating (a member of $Y$ cannot omit all of $\left\{x_{1}, x_{2}, x_{3}\right\}$. To show that $\gamma_{c}(G)=4$, suppose that $S$ is a smaller connected dominating set. Note that each $Z_{A}$ is an independent set. Also, the vertices of $Z_{A}$ have no common neighbor except in $X$, due to the requirement of distinct subscripts for edges of types (3) and (4).

If $S \cap X=\emptyset$, then $x$ is undominated or $G[S]$ is disconnected. If $S \subseteq X$, then since $G[X]$ is a 6 -cycle and $S$ must be a connected dominating set in it, $|S| \geq 4$. If $|S \cap X| \leq 2$, then $S \cap X$ does not dominate the vertices of any $Z_{A}$ such that $A \subseteq X-S$. No single vertex outside $X$ dominates $Z_{A}$, so this eliminates the cases $|S \cap X|=2$ and $x \in S$. Hence $|S \cap X|=1$ and $x \notin S$.

Let $A$ be the member of $Y^{\prime}$ not containing the vertex of $S \cap X$. Additional vertices outside $X$ are needed to dominate all of $Z_{A}$, which has neighbors outside $X$ only via edges of type (4). For each pair $B, B^{\prime} \in Y-Y^{\prime}$, there is an index $s$ such that $f_{A}\left(C_{s}^{A}\right)=\left\{B, B^{\prime}\right\}$. Since $C_{s}^{A}$ has no neighbors in $Z_{B} \cup Z_{B^{\prime}}$, there is no way to choose two vertices outside $X$ that together will dominate all of $Z_{A}$.

Finally, we show that $\gamma_{c}(\bar{G})=4$. If $y \in Z_{3456}, z \in Z_{1256}$, and $w \in Z_{1234}$, then $\{x, y, z, w\}$ is a connected dominating set of $\bar{G}$. Suppose that $S$ is a smaller connected dominating set; we speak of adjacency and domination in $\bar{G}$. Let $X^{\prime}=X \cup\{x\}$. If $S \subset X^{\prime}$, then $x$ is undominated or $\bar{G}[S]$ is disconnected.

For distinct $B, B^{\prime} \in Y-Y^{\prime}$, each vertex in $Z_{B}$ dominates one vertex in $Z_{B^{\prime}}$ and $1 / 6$ of the vertices in $Z_{A}$ for $A \in Y^{\prime}$. For distinct $A, A^{\prime} \in Y^{\prime}$, each vertex $z \in Z_{A}$ dominates all of $Z_{A^{\prime}}$ and all of $Z_{B} \cup Z_{B^{\prime}}$ such that $z \in C_{s}^{A}$ and $f_{A}\left(C_{s}^{A}\right)=\left\{B, B^{\prime}\right\}$, but only one vertex in other sets indexed by $Y-Y^{\prime}$.

Hence three vertices outside $X^{\prime}$ cannot dominate $\bar{G}$. If $S$ has two vertices $y$ and $z$ outside $X^{\prime}$, then at least two vertices of $X$ are undominated by $\{y, z\}$, and we cannot use $x$ to dominate them. Hence some $x_{i} \in S$. For all $A$ containing $x_{i}$, the job of dominating $Z_{A}$ is left to $\{y, z\}$. As discussed above, they cannot do it.

If $S-X^{\prime}=\{y\}$, then let $\left\{x_{i}, x_{j}\right\}=N_{\bar{G}}(y) \cap X$. Since $\bar{G}[S]$ is connected, we may assume that $x_{i} \in S$. Since $x_{i-1}$ and $x_{i+1}$ cannot both be $x_{j}$, we need another vertex to dominate one of them. Since $x$ dominates none of $X$, we cannot use it. Hence $S=\left\{y, x_{i}, x_{j^{\prime}}\right\}$. Now the set $Z_{A}$ with $x_{i}, x_{j^{\prime}} \in A$ and $y \notin Z_{A}$ is not dominated.

The construction in Theorem 6 has almost 15,000 vertices. We pose the problem of finding the smallest graph $G$ with $\delta^{*}(G)=6$ such that equality holds in the bound of Theorem 5 . Next we show that equality can hold in this bound only when $\delta^{*}(G)=6$.

Theorem 7. If $G$ and $\bar{G}$ are connected graphs with $\gamma_{c}(G), \gamma_{c}(\bar{G}) \geq 4$ and $\gamma_{c}(G)+$ $\gamma_{c}(\bar{G})=\delta^{*}(G)+2$, then $\delta^{*}(G)=6$.

Proof. As noted in the proof of Theorem 5, equality in the bound $\gamma_{c}(G)+\gamma_{c}(\bar{G}) \leq$ $\delta^{*}(G)+2$ requires $\left\{\gamma_{c}(G), \gamma_{c}(\bar{G})\right\}=\{4,5\}$ or $\gamma_{c}(G)=\gamma_{c}(\bar{G})=4$. In the latter case, equality requires $\delta^{*}(G)=6$. In the former case, equality requires $\delta^{*}(G)=7$. Hence it suffices to show that $\left\{\gamma_{c}(G), \gamma_{c}(\bar{G})\right\}=\underset{6}{\{4,5\}}$ with $\delta^{*}(G)=7$ is impossible. 
By symmetry, we may assume that $\delta(G)=7$. We consider the cases $\gamma_{c}(G)=4$ and $\gamma_{c}(G)=5$ together almost until the end. Let $x, X$, and the sets $T_{0} \ldots, T_{k}$ and $S_{0}, \ldots, S_{k-1}$ be as defined in the proof of Theorem 1. Note that $|N(x)|=\delta(G)=7$. Imposing equality in the computation of Equation 1 yields $k=\gamma_{c}(\bar{G})-2$ and $\left|S_{0}\right|=$ $\cdots=\left|S_{k-1}\right|=5-k=\gamma_{c}(G)-2$. Let $H=G[N(x)]$.

If two vertices in $N(x)$ dominate $X$, then they combine with $x$ to form a connected dominating set of $G$, contradicting $\gamma_{c}(G) \geq 4$. Hence the vertex set of any component of $H$ with at most two vertices combines with a vertex $z \in X$ that it does not dominate to form a connected dominating set in $\bar{G}$. Since $\gamma_{c}(\bar{G}) \geq 4$, every component of $H$ thus has at least three vertices. Since $|N(x)|=7$, this implies that $H$ has a connected subgraph $H^{\prime}$ with $\gamma_{c}(G)-1$ vertices; let $U=V\left(H^{\prime}\right)$ and $W=N(x)-U$.

From $\left|S_{0}\right|=\gamma_{c}(G)-2$, we conclude that $U$ dominates $X$. If $U$ dominates $W$, then $U \cup\{x\}$ is a connected dominating set with size less than $\gamma_{c}(G)$. Hence we may choose $z^{\prime} \in W$ such that $z^{\prime}$ has no neighbor in $W$. We complete a contradiction by building a connected dominating set in $\bar{G}$ of size $|W|$.

If $\gamma_{c}(\bar{G})=4$, then no three vertices in $N(x)$ can dominate $X$, since $\gamma_{c}(G)=5$. Hence we may choose $z \in X-N(W)$. Now $\left\{x, z, z^{\prime}\right\}$ is a connected dominating set in $\bar{G}$, contradicting $\gamma_{c}(\bar{G})=4$.

If $\gamma_{c}(\bar{G})=5$, then no two vertices in $N(x)$ can dominate $X$, since $\gamma_{c}(G)=4$. Hence for any partition of $W$ into two pairs, we can find vertices $z_{1}, z_{2} \in X$ that are common nonneighbors for the vertices in the pairs. Now $\left\{x, z_{1}, z_{2}, z^{\prime}\right\}$ is a connected dominating set in $\bar{G}$, contradicting $\gamma_{c}(\bar{G})=5$.

\section{A BOUND FOR $\gamma_{c}(G) \gamma_{c}(\bar{G})$}

We conclude with a sharp upper bound for $\gamma_{c}(G) \gamma_{c}(\bar{G})$. In order to complete the characterization of when equality holds, we will use the following technical lemma to eliminate unwanted possibilities for equality among graphs with eight vertices.

Lemma 8. There is no 4-regular 8-vertex graph $G$ with $\gamma_{c}(G)=3$ and $\gamma_{c}(\bar{G})=4$.

Proof. Let $G$ be such a graph, if one exists; we restrict its properties. Every edge of $G$ lies in a triangle, since otherwise its endpoints would form a connected dominating set. Hence $G$ has no 4-clique, since the edges leaving it would not lie in triangles.

For a partition of $V(G)$ into sets of size 5 and 3, at least six edges join the two parts, since $G$ is 4-regular. Hence no vertex neighborhood induces a 4-cycle. If some edge $R$ lies in three triangles, then the three vertices in triangles with it form an independent set $S$, the edges from $S$ to the remaining three vertices form a 6-cycle, and those three vertices form a triangle $T$. The resulting graph $G_{1}$ is unique and appears on the left in Figure 2, but $\gamma_{c}\left(\bar{G}_{1}\right)=3$.

Given an arbitrary vertex $x$, let $U=V(G)-N[x]$; note that $|U|=3$. If every edge of $G$ lies in only one triangle, then eight edges join $N[x]$ to $U$. Some vertex of $U$ receives at least three of these. Two of those edges come from one of the triangles containing $x$, yielding an edge in two triangles.

Hence some $x$ has an incident edge in two triangles. Since we have no edges in 0 or 3 triangles, $N(x)$ induces a path. Hence exactly six edges join $N(x)$ to $U$, and $U$ is a triangle. The endpoints $y$ and $z$ of the path induced by $N(x)$ each have two 
neighbors in $U$. If $u, u^{\prime} \in N(y) \cap N(z)$, then edge $u u^{\prime}$ is in three triangles and the graph is $G_{1}$ again. Otherwise, the graph is $G_{2}$, shown in Figure 2.

As shown in Figure $2, \gamma_{c}\left(\bar{G}_{1}\right)=\gamma_{c}\left(\bar{G}_{2}\right)=3$. We have eliminated all cases.
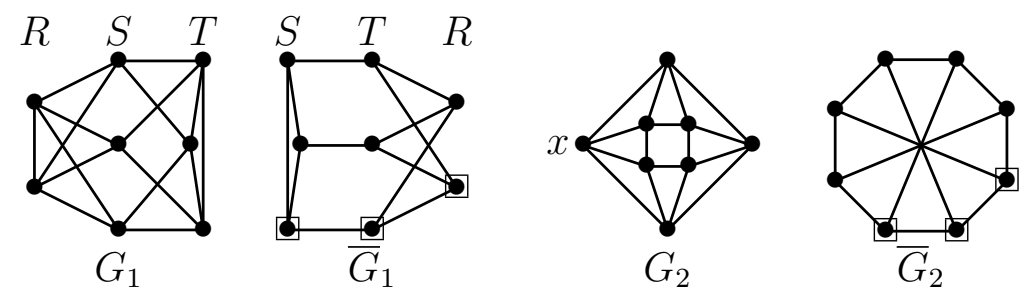

Figure 2. The graphs $G_{i}$ of Lemma 8 and their complements

Theorem 9. If $G$ and $\bar{G}$ are connected $n$-vertex graphs with $n \geq 7$, then $\gamma_{c}(G) \gamma_{c}(\bar{G}) \leq$ $2 n-4$, with equality only when $G$ or $\bar{G}$ is a path or a cycle. The bound fails for $C_{5}$ and for one pair of complementary 6-vertex graphs.

Proof. For a path or a cycle with at least 6 vertices, $\gamma_{c}(G)=n-2$ and $\gamma_{c}(\bar{G})=2$; hence the bound is sharp. Since $\gamma_{c}\left(C_{5}\right)=\gamma_{c}\left(\bar{C}_{5}\right)=3$, the bound fails for $C_{5}$. The same is true for the 6-vertex graph obtained by subdividing one edge of $K_{4}$ twice.

Now consider the upper bound. Since $G$ and $\bar{G}$ are connected, $\gamma_{c}(G), \gamma_{c}(\bar{G}) \geq 2$. By symmetry, we may assume that $\gamma_{c}(G) \leq \gamma_{c}(\bar{G})$. If $\gamma_{c}(G)=2$, then $\gamma_{c}(\bar{G}) \leq n-2$ yields $\gamma_{c}(G) \gamma_{c}(\bar{G}) \leq 2 n-4$. Equality requires that $\bar{G}$ has no spanning tree with more than two leaves; this happens only when $\bar{G}$ is a path or a cycle.

For $\gamma_{c}(G) \geq 4$, we rewrite Theorem 1 to isolate $\gamma_{c}(G) \gamma_{c}(\bar{G})$ and then apply Theorem 5 and the fact that $n \geq 4$ when $G$ and $\bar{G}$ are connected to compute

$$
\begin{aligned}
\gamma_{c}(G) \gamma_{c}(\bar{G}) & \leq \delta^{*}(G)-5+2\left[\gamma_{c}(G)+\gamma_{c}(\bar{G})\right] \\
& \leq 3 \delta^{*}(G)-1 \leq 3 \frac{n-1}{2}-1<2 n-4
\end{aligned}
$$

Hence we may assume that $\gamma_{c}(G)=3$ and $\gamma_{c}(\bar{G}) \geq 3$. This requires $\operatorname{diam}(G)=$ $\operatorname{diam}(\bar{G})=2$, and hence $\gamma_{c}(\bar{G}) \leq \delta(\bar{G})+1$. Note also that $\delta^{*}(G) \geq 2$.

If $\delta(\bar{G})=2$, then $\gamma_{c}(G) \gamma_{c}(\bar{G}) \leq 9$, which suffices when $n \geq 7$. If $\delta(\bar{G}) \geq 4$, then Theorem A yields $\gamma_{c}(\bar{G}) \leq(3 n-8) / 5$; always $3(3 n-8) / 5<2 n-4$ for positive $n$.

This leaves the case $\delta(\bar{G})=3$ and $\gamma_{c}(\bar{G})=4$, so $\gamma_{c}(G) \gamma_{c}(\bar{G})=12$. Since $12<2 n-4$ when $n>8$, we are left with $n \in\{7,8\}$.

To eliminate $n=7$ we use Theorem B. Since $\gamma_{c}(\bar{G}) \leq n-\Delta(\bar{G})$, having $\gamma_{c}(\bar{G})=4$ requires $\Delta(\bar{G}) \leq 3$. Hence $\bar{G}$ is 3 -regular with 7 vertices, but no such graph exists.

This leaves graphs with 8 vertices. Each vertex of $\bar{G}$ has degree 3 or 4 (again by Theorem B), and we have $\gamma_{c}(\bar{G})=4, \gamma_{c}(G)=3$, and $\operatorname{diam}(\bar{G})=\operatorname{diam}(G)=2$. Let $x$ be a vertex of maximum degree in $\bar{G}$, and let $U=V(G)-N[x]$. If $x$ has degree 4 , then $|U|=3$. If any neighbor of $x$ has two neighbors in $U$, then $\gamma_{c}(\bar{G}) \leq 3$, since $\operatorname{diam}(\bar{G})=2$. Hence at most four edges join $U$ to $N(x)$. This leaves degree-sum at least 5 for edges within $U$, so $G[U]$ is a triangle. Now $\gamma_{c}(\bar{G}) \leq 3$, using $x$, a vertex of $N(x)$ having a neighbor in $U$, and that neighbor.

Hence we may assume that $\bar{G}$ is 3-regular and $G$ is 4-regular. By Lemma 8, there is no such graph with $\gamma_{c}(G)=3$ and $\gamma_{c}(\bar{G})=4$. 


\section{ReFERENCES}

[1] Y. Caro, R. Yuster, and D. B. West, Connected domination and spanning trees, SIAM J. Discrete Math. 13 (2000), 202-211.

[2] J. E. Dunbar, T. W. Haynes and S. T. Hedetniemi, Nordhaus-Gaddum bounds for domination sum in graphs with specified minimum degree, Util. Math. 67 (2005) 97-105.

[3] J. R. Griggs and M. Wu, Spanning trees in graphs of minimum degree 4 or 5, Discrete Math. 104 (1992), pp. 167-183.

[4] S. T. Hedetniemi and R. C. Laskar, Connected domination in graphs, In B. Bollobás, editor, Graph Theory and Combinatorics. Academic Press, London, 1984.

[5] J. P. Joseph and S. Arumugam, Domination in graphs, Internat. J. Management Systems 11 (1995) 177-182.

[6] F. Jaeger and C. Payan, Relations du type Nordhais-Gaddum pour le nombre d'absorption d'un graphe simple, C. R. Acad. Sci. Paris Sér. A 274 (1972) 728-730.

[7] D.J. Kleitman and D.B. West, Spanning trees with many leaves, SIAM J. Discrete Math. 4 (1991), pp. $99-106$.

[8] E. A. Nordhaus and J. W. Gaddum On complementary graphs, Amer. Math. Monthly 63 (1956), pp. 175-177.

[9] E. Sampathkumar and H.B. Walikar, The connected domination number of a graph, J. Math. Phys. Sci. 13(6) (1979), pp. 607-613. 